\title{
Tuberculosis-immune reconstitution inflammatory syndrome in HIV: From pathogenesis to prediction
}

Article in Expert Review of Clinical Immunology · March 2014

DOI: 10.1586/1744666X.2014.892828 · Source: PubMed

CITATIONS

6

3 authors, including:

\section{Narendran Gopalan}

Indian Council of Medical Research

54 PUBLICATIONS 957 CITATIONS

SEE PROFILE
Soumya Swaminathan

Indian Council of Medical Research

397 PUBLICATIONS 11,117 CITATIONS

SEE PROFILE

Some of the authors of this publication are also working on these related projects: 


\section{EXPERT REVIEWS}

Tuberculosis-immune reconstitution inflammatory syndrome in HIV: from pathogenesis to prediction

Expert Rev. Clin. Immunol. Early online, 1-15 (2014)
Narendran Gopalan ${ }^{1}$, Bruno Bezerril Andrade ${ }^{2}$ and Soumya Swaminathan ${ }^{* 1}$

${ }^{1}$ National Institute for Research in Tuberculosis, 1, Mayor Sathyamoorthy Road, Chetpet, Chennai 600 031, India ${ }^{2}$ Immunology Section, laboratory of Parasitic Diseases, National Institute of Allergy and Infectious Diseases, National Institutes of Health, Bethesda, MD 20892-8003, USA

*Author for correspondence:

Tel.: +914428369700

Fax: +914428362528

doctorsoumya@yahoo.com

Tuberculosis-immune reconstitution inflammatory syndrome (TB-IRIS) is an exaggerated, dysregulated immune response against dead or viable antigens of Mycobacterium tuberculosis that frequently occurs after initiation of antiretroviral therapy despite an effective suppression of HIV viremia. Scientific advances in IRIS pathogenesis have led researchers and clinicians to postulate risk factors that could possibly predict this syndrome, in an attempt to reduce the incidence and the severity of IRIS, with appropriate anti-inflammatory therapy. This review is a summary of the available literature on pathogenic mechanisms involved from the macro to the micro level, the clinical spectrum, available predictors and the scope of these biomarkers to function as specific therapeutic targets, that could effectively modulate or ameliorate this syndrome in future.

KEYWORDS: ART • biomarkers for IRIS $\bullet$ HAART $\bullet$ HIV •IRIS • paradoxical reaction • TB

Tuberculosis (TB) is the commonest opportunistic infection (OI) in HIV-infected patients, causing the highest casualties worldwide [1]. An interesting phenomenon that has emerged with the early initiation of highly active antiretroviral therapy (HAART), concomitantly with specific treatment like anti-TB therapy (ATT) in coinfected patients, is called immune reconstitution inflammatory syndrome (IRIS) [2]. IRIS is the paradoxical worsening of an existing lesion/ condition or unmasking of a previously quiescent lesion after HAART initiation. This dysregulated augmentation of immune responses occurs despite an adequate therapeutic response to antiretroviral therapy, as evidenced by effective virological suppression [3-6]. Although IRIS is associated with a number of infectious and noninfectious conditions, TB-associated IRIS (TB-IRIS) tops the list as the most frequent cause of systemic IRIS among HIV-positive patients starting HAART $[5,6]$. A similar phenomenon of 'paradoxical reaction' was reported in $2 \%$ of HIV-negative individuals treated for tuberculomas of the brain and $\mathrm{TB}$ lymph nodes. [7,8]. Among HIV-positive individuals with active TB, TB-IRIS incidence ranges from 8 to $43 \%[2,5,7,8]$ and could reach up to $54 \%$ [4] depending on the epidemiological setting.

IRIS also occurs after abrupt withdrawal of anti-inflammatory drugs like cyclosporine, TNF- $\alpha$ blockers, natalizumab and steroids in HIV-negative patients with autoimmune diseases, which implies that a common mechanism exists. Restoration of pathogen-specific immune responses, succeeding long-standing immunesuppression, appears to be the cause [9-11]. IRIS is usually self-limiting and manageable with anti-inflammatory drugs, and mortality is around 3\% [2]. However, neurological-IRIS, which constitutes $10-12 \%$ of overall IRIS cases, carries a higher mortality of $13 \%$ and requires aggressive steroid therapy [12]. This review summarizes the various scientific concepts in IRIS including clinical spectrum, attributable risk factors, possible predictors, available interventions and future exploratory targets that could benefit a HIV patient with uneventful immune recovery.

\section{Types of TB-IRIS}

TB-IRIS syndrome is of two types: 'paradoxical' and 'unmasking' IRIS. Paradoxical TB-IRIS occurs after an initial improvement with 
pathogen-specific therapy (ATT), followed by an apparent deterioration after HAART initiation [5]. At the time of IRIS event, smear for acid-fast bacilli may be positive; however, cultures are invariably negative. Nonviable organisms or residual antigens trigger the reaction [9]. Occasionally, when IRIS occurs within a short span of time after ATT initiation, the specimen could still yield a positive culture, but with a lower grade at IRIS time point, compared with baseline. The second type, the unmasking variety, is caused by viable Mycobacterium tuberculosis (MTB) that could result from a real unmasking of an occult disease or failure to diagnose the disease prior to HAART initiation [1]. In this situation, the immune response is unable to completely contain the infection. This can progress to overt disease and needs to be addressed as if dealing with a new episode of TB [5,9].

\section{Diagnostic criteria for recognition of IRIS}

Criteria for IRIS diagnosis have evolved over the years to especially suit resource-limited developing countries, where the magnitude of this problem is greater. This obviated the need for facilities and expertise, such as virological assays and biomarker evaluation, which form the benchmark for IRIS diagnosis. French et al. [13] in 2004 defined IRIS as an atypical manifestation of an OI with a decline in viral load by at least one $\log _{10}$ HIV RNA copies per $\mathrm{ml}$ as the major criterion. Minor criteria included a rise in $\mathrm{CD} 4$ count, augmentation of specific immune responses and spontaneous resolution without specific antimicrobial therapy. The simplified definition by Shelburne et al. included a reduction in viral load, an increase in CD4 $\mathrm{T}$ cell count, with clinical signs and symptoms not associated with the expected course of OI after ruling out drug toxicity [3]. More recently, radiological deterioration, assured compliance to therapy and a positive tuberculin skin testing (TST) were added to the existing criteria [14]. In 2008, The International Network for the Study of HIV-associated IRIS (INSHI) published an official consensus case definition to include both types of IRIS. This eliminated the need for viral load estimation and biological marker evaluation, thereby facilitating decentralization of the ART program [5]. At least one major and/or two minor (INSHI) criteria are needed to fulfill to establish the diagnosis of IRIS. The scientific justification by the INSHI group for excluding viral load and CD4 estimation from the diagnostic criteria was that plasma viral load would decrease in all patients adhering to HAART, irrespective of IRIS occurrence [5]. Increase in CD4 T cells usually follow a lag period and this increase may not be apparent at the time of IRIS $[13,15]$.

Unmasking IRIS, as per INSHI guidelines, included ATTnaïve patients with active TB diagnosed after HAART initiation due to a marked inflammatory component occurring within 3 months of anti-HIV treatment. The time frame of 3 months was introduced as it coincided with the acute recovery stage when proinflammatory responses would operate at the zenith [5].

\section{Incidence \& clinical spectrum}

Various cohort studies focusing specifically on TB-IRIS and their characteristics are listed in TABLE 1. A common observation is that the shorter the ATT-HAART interval, the greater is the incidence and intensity of TB-IRIS. Symptoms and signs of TB-IRIS differ with respect to the stage of the disease, site of lesion and its dissemination [16,17]. Clinical manifestations vary from localized lesions like isolated peripheral lymphadenopathy and subcutaneous abscesses to severe forms like acute respiratory distress syndrome (ARDS) and viscus perforation, which may end fatally [14]. Neurological-IRIS is particularly lethal, manifesting as meningitis, space-occupying lesions, radiculomyelitis and spondylitis. Lesions may be multiple with extensive perilesional edema [18]. Lymphadenopathy-causing obstruction, osteomyelitis, subcutaneous abscesses and thromboembolic episodes have also been reported [19]. Serositis is not infrequent [20]. Abdominal manifestations include hepatosplenomegaly, psoas abscesses, splenic microabscesses and rupture, epididymo-orchitis, ureteral compression and acute renal failure [21]. Hypercalcemia has been reported, due to secretion of 1,25-dihydroxy cholecalciferol by activated macrophages, during the IRIS episode [22].

A prospective study among HIV patients with cultureconfirmed rifampicin-sensitive newly diagnosed pulmonary $\mathrm{TB}$, started on ART (at a median interval of 20 days after ATT), showed the IRIS occurrence to be 54\% (26 of 48), occurring in a median period of 10 days (interquartile range 7-16) [4]. At enrollment, extrapulmonary focus of TB in the form of peripheral and mediastinal lymphadenopathy was present in 57\% of the IRIS cases [4]. Fever with rigor or chills resembling malaria was the commonest symptom followed by radiological deterioration [4]. Sometimes, radiological worsening occurred without clinical deterioration, called radiological or cryptic IRIS [4,17]. Lymph node enlargement, as a manifestation of IRIS, occurred in $75 \%$ of cases with or without intrathoracic adenopathy (20\%). The median time to clinical resolution was 13 days (interquartile range 9-23) [4].

\section{Clinical risk factors for IRIS occurrence}

Risk factors may be pathogen or host related or may be caused by interplay of both (Figure 1). Risk factors consistently associated with IRIS include low CD4 $\mathrm{T}$ cell counts at baseline [2,5,23], low BMI [24], presence of extrapulmonary TB [1,5], other OIs [15], shorter ATT-ART interval [4] and substantial increase in CD4 T cell count or rapid drop in viral load $[4,5,15,23,24]$. Patients with advanced stage of HIV constitute the most vulnerable group for IRIS occurrence, as they need ART to be started concomitantly with ATT. Higher risk of IRIS occurrence has been reported among patients initiating ART within the first 2 months of starting ATT [25,26]. Haddow et al. [27] showed the progressive decline in IRIS incidence to be 17\% (95\% CI: 6-26\%), with a $43 \%$ reduction in mortality, for every 50 cell increase in nadir CD4. Our own experience has shown the incidence of TB-IRIS in patients with baseline CD4 of $<50$, 50-99, 100-199 and $>200$ cells $/ \mathrm{mm}^{3}$ to be $72,63,52$ and 


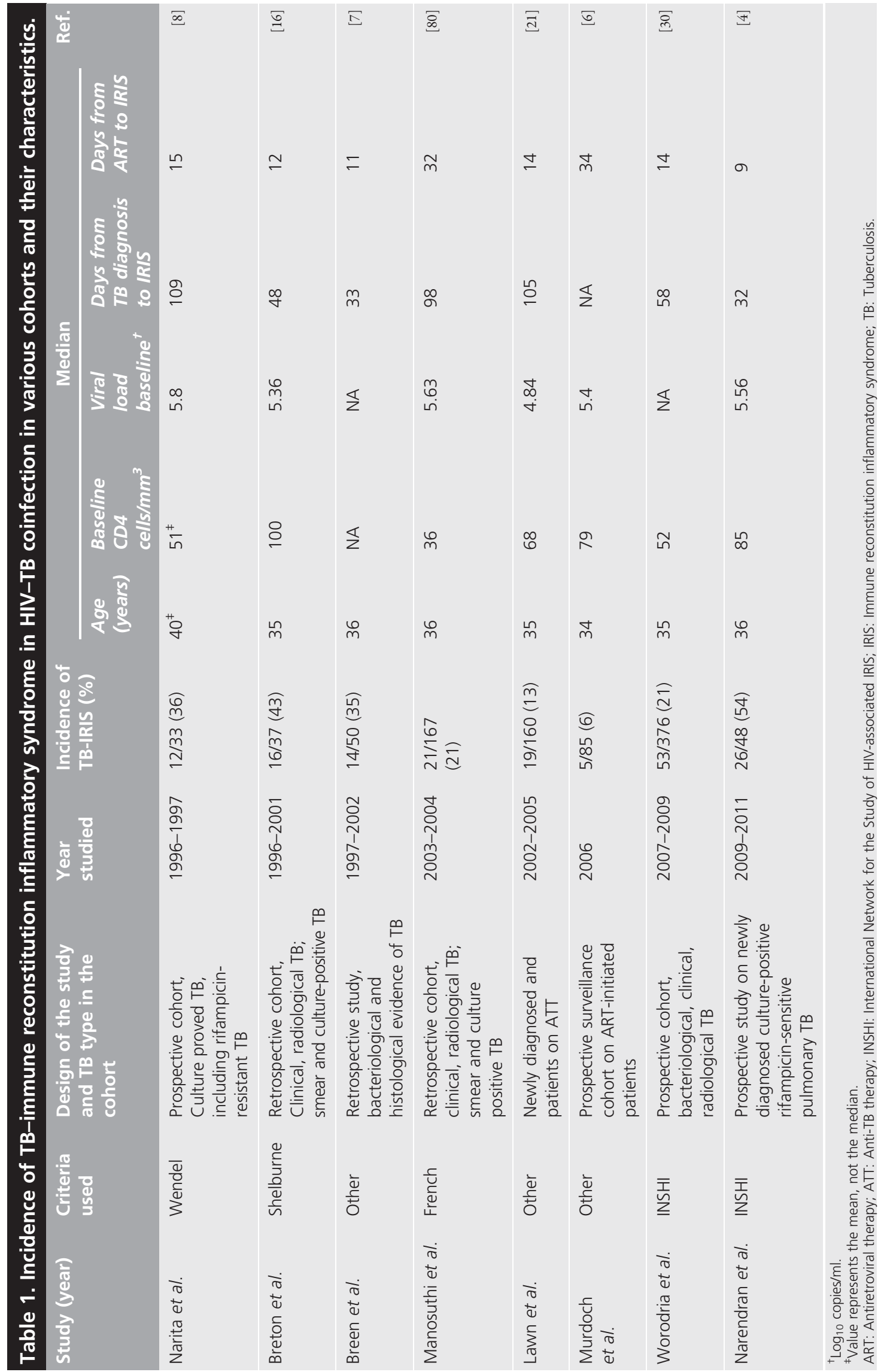



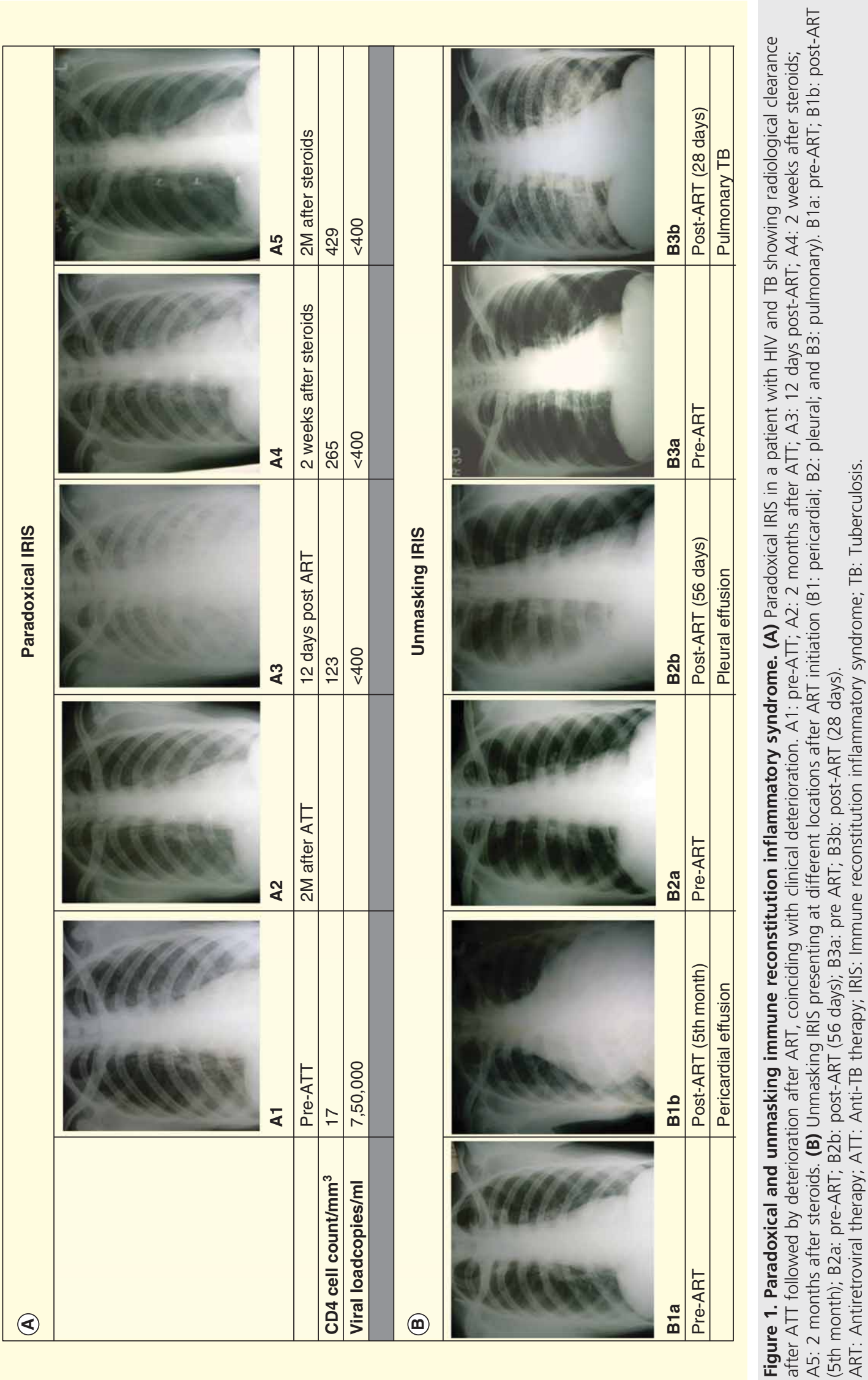
$20 \%$, respectively [4]. The other predisposing factors include low levels of hemoglobin, hematocrit and CD4/CD8 ratio [4,5,28-30]. Patients with immune restoration disease arising from mycobacterial infection rarely carried IL6- $174^{*} \mathrm{C}$ (36 vs $61-71 \%$ ) and never carried TNFA-308*2 (0 vs $23-52 \%$ ), making genetic predisposition a causal factor [31]. A boosted protease inhibitor regimen was also found to be an independent risk factor of IRIS due to its potent viral suppression [15].

\section{Optimal timing of ART initiation \& IRIS}

The interim results of the Starting ART at three-time points in TB trial showed a clear advantage of initiating ART within the treatment period of $\mathrm{TB}$, reducing mortality by $56 \%$ compared with delaying ART till ATT completion [25]. However, comparing patients who started ART within the first month of ATT with those who started at the end of 2 months, a three-times higher incidence of IRIS and a 90\% higher incidence of toxicity was found, without any additional survival benefits [25,32]. Patients with CD4 cell count $<50$ cells alone benefited by the immediate initiation of ART, as evidenced by a one-third reduction in mortality [32]. Havlir et al. [33] conducted a similar study (ACTG 5221), but with a composite endpoint combining mortality and AIDS-defining illness and found that the advantage of early ART initiation within 2 weeks of starting ATT was evident only in the group of patients with CD4 $<50 \mathrm{cells} / \mathrm{mm}^{3}$. The Cambodian Early versus Late Introduction of Antiretrovirals study, with a median CD4 of 25 cells $/ \mathrm{mm}^{3}$ among study subjects, mirrored the same results as that of Havlir et al. with a greater reduction in mortality in the early ART initiation group [26,33]. The retrospective multicentric cohort of patients with HIV infection in the Madrid South Eastern Metropolitan Crown (COMESEM) study also demonstrated survival benefits when ART was started within the intensive phase of ATT [34]. Among studies done on HIV-TB patients at the National Institute for Research in TB at Chennai, India, an overall mortality of $14.7 \%$ was observed when ART was started at the end of 2 months compared with $8.3 \%$ when ART was initiated within the intensive phase of ATT $(\mathrm{p}=0.07)$. However, the incidence of IRIS was $20 \%$ when ART was started at 2 months of ATT compared with $38 \%$ when started within the intensive phase $(p=0.01)$ [20,35]. The unified conclusion from various studies conducted in different settings is that among patients with CD4 <50 cells, immediate initiation of ART within 2 weeks is beneficial in reducing mortality and other AIDS-defining illnesses in this cohort. However, in patients with CD4 >50 cells, cautious deferral of ART to the first few weeks of ATT helps in reducing toxicity and IRIS, without compromising on survival benefits. This formed the basis for WHO to recommend initiation of ART between 2 and 8 weeks of ATT [36,37]. Starting ART when the CD4 count is high may also serve as a preventive strategy against IRIS.

\section{Immunopathogenesis of IRIS}

Pathogenesis of IRIS is multifactorial, involving a number of interrelated immune mechanisms. With MTB being a chronic infection, antigens of TB bacilli persist in tissues even after effective ATT, inciting IRIS once immunity is restored [1]. In addition, treatment of TB itself can enhance immunity causing IRIS [3]. TB-IRIS is associated with renewed vigor for granuloma formation and suppuration due to intense inflammatory reaction caused by IRIS, along with TST conversion to positivity $[1,38]$.

\section{What triggers IRIS (from retrovirus to cytokines)? Rapidity of viral load decline}

Earlier studies on IRIS concluded that a dramatic reduction in viral load coupled with a rapid increase in circulating CD4 lymphocytes after HAART triggered IRIS. The study by Manabe et al. [15] showed that the risk of IRIS increased by 2.43-times (1.00-5.96; $\mathrm{p}=0.05)$ when HIV-1 RNA declined by $2.5 \log _{10}$ copies $/ \mathrm{ml}$.

\section{Increase in number of circulating lymphocytes after HAART}

CD4 T cells, the main target of HIV virus, get progressively depleted by various cell-mediated mechanisms involving macrophages, NK cells and CD8 T cells apart from apoptosis. In the phase of persistent HIV viremia, there is no replenishment of CD4 $\mathrm{T}$ cells by either production or redistribution. Chronic infections such as HIV and TB have a direct negative impact on the bone marrow, limiting CD4 recovery [39]. Even a modest reduction in viral load is enough to regenerate $\mathrm{T}$ cell production and redistribution [40]. Interestingly, absolute increase in CD4 T cell was not associated with increased risk of IRIS [15]. Hence, viral load decline or CD4 increase alone may not be sufficient to accurately predict IRIS occurrence [27]. Considerable variations exist in the rate of reconstitution of CD4 lymphocytes upon HAART initiation. Severe CD4 T lymphocytopenia, higher age and longer interval between HIV diagnosis and treatment may lead to immunological discordance, with continued low levels of CD4 T cells even after ART initiation, despite effective virological suppression. These patients are unlikely to develop IRIS, and prognosis is not that satisfactory [39].

\section{Role of $T$ cell subsets \\ $\mathrm{CD}^{+} \mathrm{T}$ cells}

CD4 $\mathrm{T}$ cell reconstitution follows a bimodal pattern. There is an initial redistribution of $\mathrm{CD} 4+\mathrm{CD} 45 \mathrm{RO}+$ cells. With an effective suppression of HIV viremia, it takes a few months for de novo synthesis of CD4+CD45RA+ cells expressing Ki67 that contributes to a sustained rise $[15,39,41]$. The majority of paradoxical IRIS, occurring within the first 3 months of ART initiation, is due to the increase in CD45RO+ effector memory cells. Redistribution of these cells augments antigen-specific responses as they gain access to the site of infection and trigger an inflammatory reaction [14]. Early data suggested that IRIS occurs due to an increase in the number of activated CD4 $\mathrm{T}$ cells, inducing an exaggerated Th1 type of immune 
response. The functional restoration was much more important for causing IRIS than mere increase in the number of T cells [17,38,42].

More recently, Antonelli et al. [43] studied the relative number of activated $\mathrm{T}$ cells, Tregs and exhausted cells and their role in IRIS occurrence. In that study, the HIV-RNA levels, CD4 and Treg numbers were similar in both the IRIS and non-IRIS groups (patients who did not experience IRIS) at all time points including at the time of IRIS event. Subgroup analysis revealed that there was an isolate in CD4 $\mathrm{T}$ effector memory cells in the IRIS group at IRIS event compared with non-IRIS patients, but who possessed a higher CD4. At the corresponding time point, it was the naïve CD4 $\mathrm{T}$ cells that predominated among the non-IRIS group. These findings implied that the non-IRIS patients reconstituted their naïve $T$ cell compartment earlier than the IRIS group, whereas the IRIS patients retained a higher frequency of effector memory CD4 T cells at IRIS event. Effector memory CD4 $\mathrm{T}$ cells displaying both replication and activation markers were shown to be the hallmark of IRIS syndrome [43]. This signifies effective reconstitution and activation after ART that is retained for a longer period in IRIS patients than non-IRIS patients. It is probable that the prolonged survival of these activated cells may be facilitated by sustained antigenic persistence. The trend was reversed in non-IRIS patients with more central memory CD4 $\mathrm{T}$ cells than in IRIS patients at 6 months in the same study [43]. IRIS patients demonstrated a higher fraction of polyfunctional IFN- $\gamma$ cells during IRIS episode and a large proportion of T-effector cells [38].

\section{Role of $\mathrm{CD}^{+} \mathrm{T}$ cells}

An absolute increase in CD8 memory cells was observed in IRIS patients before ART initiation [43]. Markers of active replication (CD38, Ki67 and HLADR) were higher at 6 months in the IRIS group with a lower frequency of CD57-GrB+expresssing CD8 cells at baseline [43]. Antonelli et al. also demonstrated a higher frequency of CD8 central memory $\mathrm{T}$ cells among non-IRIS patients, but at 6 months post-ART initiation. Espinosa et al. [44] found an overall increase in CD8 $\mathrm{T}$ cells where a selective expansion to MTB antigens occurred, just preceding IRIS. CD8 T cells (CD38+, HLA-DR+) showed an expansion accompanied by slow decay in 12 weeks coinciding with the onset of TB-IRIS. CD38+HLA-DR + activated $\mathrm{CD}^{+} \mathrm{T}$ cells are a marker of chronic HIV progression, and HAART is supposed to bring down the number of cells. It is not clear whether the increase in the specific phenotype CD38+HLADR $+\mathrm{CD}^{+}$is the cause or the effect of IRIS [43]. Mahnke et al. [45] longitudinally studied the changes occurring in CD4 and CD8 T cell population during ART and subsequent IRIS (including TB-IRIS) and found that there was an overall reconstitution of $\mathrm{T}$ cells during ART. This was not confined to the IRIS group alone. However, CD8 programmed death-1(PD-1+) cells were more frequent in the IRIS group along with lower $\mathrm{CD} 28^{+}$and $\mathrm{CD} 127^{+}$
T cells. CD8 cells of the IRIS group demonstrated delayed recovery of $C D 28$ and $C D 127$ expression, which could be the result rather than the cause of IRIS. Increased expression of costimulatory molecules like ICOS was observed. There was also a simultaneous increase in CD152 (CTLA4) that functions to inhibit $\mathrm{T}$ cell activation by counteracting the CD28 signals, thereby terminating $\mathrm{T}$ cell responses. Lymphocyte activation gene-3 (LAG3) molecules were also more in the IRIS group before ART initiation [43,45].

\section{Role of Treg}

Tregs, a subset of CD4 T helper cells, constitute 5\% of the total population. They have been aptly described as 'committed suppressors' of the immune system by Zaidi et al. [10]. The natural Tregs expressing the transcription factor Fork head box protein 3 (Foxp3) protein function by suppressing the secretion of cytokines and proliferation of proinflammatory cells, maintaining $\mathrm{T}$ cell homeostasis. HIV induces Tregs to move to peripheral and mucosal-associated lymphoid tissue, which gets reversed by ART. The earlier concepts were that depletion [46] or delayed reconstitution of Treg-related regulatory mechanisms [47] triggered IRIS. Montes et al. [48] found that Tregs generally reconstitute like any other immune-related cell during recovery. Interestingly, Tregs were found to increase at par with CD4 $\mathrm{T}$ cells soon after ART initiation. More recently, higher frequencies of Tregs coexpressing Ki67, HLA-DR and PD-1 and PD-1 CD4 T cells expressing CTLA4, LAG3 or ICOS before ART initiation have been described as a defence mechanism, in anticipation of IRIS that may follow [43]. Meintjes et al. [49] found no significant difference in Foxp3 expression between TB-IRIS and non-IRIS groups. The expressions of activation factors such as CD71 (transferring factor) and HLA-DR were also similar in both the groups. Zaidi et al. [10] found a slower decline in Tregs in the IRIS group compared with non-IRIS group, persisting for 3 months post-ART initiation. The trends were similar whether Tregs were defined and labeled by Foxp3 alone or CD25 and Foxp3 together. This study, which included $10 \%$ of HIV-2 infection, helped to hypothesize that similar mechanisms operated in HIV-2 for IRIS occurrence. Seddiki et al. [50] added clarity to the role of Tregs in TB-IRIS. The study found a higher $\mathrm{T}$ reg population defined by CD127 lo CD25+Foxp3+ cells. This combination of markers helped to differentiate true Treg from recently activated effector cells that also possess upregulated Foxp3. The addition of Ki67 increased the specificity, which helped in confirming the source to be due to proliferation rather than altered trafficking. However, in vitro studies by the same group found Treg function to be defective even though numbers rise. Treg isolated from TB-IRIS patients failed to decrease the cytokine levels of IL-6, IFN- $\gamma$ and TNF- $\alpha$ compared with Treg from healthy controls in vitro, with higher levels of IL-7 strongly inhibiting Treg-mediated suppression. To the contrary, IL-10-induced anti-inflammatory responses were augmented when Treg from healthy controls were used in crossover assays in vitro [50]. 
Role of inhibitory molecules on T cells

Chronic infection like HIV causes $\mathrm{T}$ cell exhaustion, due to continued viral replication, leading to functional impairment. Blockade of PD-1, a marker of functional exhaustion restores HIV-specific T cell activity [51]. A study by Antonelli et al. [43] showed that PD-1+effector memory $\mathrm{T}$ cells were more frequently present in patients with IRIS before HAART initiation, irrespective of the underlying OI. In addition, the authors demonstrated the role of PD1 expression in suppressing the function of $\mathrm{T}$ lymphocytes, by production of the anti-inflammatory cytokine IL-10. After ART initiation, the process gets reversed, leading to decreased levels of IL-10, triggering the inflammatory cascade once again. In a follow-up study, Mahnke et al. [45] showed an elevated PD-1 expression in both CD4 and CD8 cells at baseline among IRIS cases (including TB-IRIS cases) compared with non-IRIS cases. Higher proportions of PD-1, along with other coexpressing inhibitory coreceptors like LAG3 and CTLA4, suggest that T cells are hyperactive but with functional impairment, which is reversed by HAARTcausing IRIS [43].

\section{Role of NK cells in IRIS}

NK cells are large granular lymphocytes, which play a vital role in graft rejection and killing of malignant and pathogeninfected cells, regulated by the cytokines IL-12, IL-15, IL-18 and IFN- $\gamma$ [52]. NK activity $\left(\mathrm{CD} 56^{+}\right.$and or $\left.\mathrm{CD} 16^{+}\right)$is a prerequisite for restricting viral replication and preventing CD4 cell depletion [53]. Pean et al. [54] studied the association of NK cells in TB-IRIS and found no significant difference in NK cell subsets obtained from patients experiencing IRIS and those who did not. However, a higher degranulation capacity (expression of $\mathrm{CD}^{\circ} 9^{+}$by NK cells) among IRIS patients, prior to ART initiation, was observed (compared with non-IRIS counterparts). However, at the time of IRIS, there was no difference in CD69 expression but lower levels of Nkp30, Nkp46 and NkG 2D suggesting a lowered inhibitory phenotype at IRIS. T cells, under the influence of IFN- $\gamma$ produced by NK cells, get activated in lymph nodes and preferentially differentiate into Th1 lineage. These activated $\mathrm{T}$ cells in turn secrete Il-12, IL-15and Il-18 that sustains the NK cell activation, forming a positive feedback loop [54].

\section{Proinflammatory mechanisms in the presence of antigenic stimulus}

IRIS can also occur upon reversal of dysfunctional $\mathrm{T}$ lymphocytes, emphasizing the need for functional restoration in addition to mere increase of numbers [55]. Severe lymphocyte depletion favored pathogenic persistence and dissemination. This leads to accumulation of antigens inciting a dysregulated immune response upon immune restoration. The antigens could be viable antigens (organisms) in the case of unmasking IRIS or predominantly dead/shed antigens as in paradoxical IRIS [9]. The better understood costimulatory pathway in $\mathrm{T}$ cell activation, involving the $\mathrm{T}$ cell surface coreceptor $\mathrm{CD} 28$, provides insight into the regulatory function of the APC-T cell coreceptor in the presence of antigens. CD28 coreceptor binds to the costimulatory molecules B7-1 (CD80) and B7-2 (CD86), expressed on activated APCs, only when antigens prevail in abundance. In this scenario, it increases B-7 binding to CD28 receptor followed by stimulation of $\mathrm{T}$ cells. On the other hand, when there is a lower level of antigens, CTL4 and PD-1 binding to B-7 are pronounced leading to anergy. This serves as an autoregulation mechanism against IRIS occurrence and also signifies the need for a minimum threshold of antigenimia above which IRIS occurs. This was confirmed by the Barber et al. mouse model, where neither situation resulted in IRIS viz. when the cell was fully functional with effective and timely antigenic clearance, and when the dysfunctional $\mathrm{T}$ cells were loaded with viable or dead antigens but unable to mount an inflammatory response [11].

Irrespective of the pathogen involved, Mahnke et al. [45] suggested an upgrading reaction, caused by pathogen-specific polyfunctional cells, targeting the specific pathogen inciting the reaction. Cross-sectional analysis among TB-IRIS and non-IRIS groups by Meintjes et al. [49] showed higher frequencies of MTB antigen-specific IFN- $\gamma$-secreting T cells that induced the 'cytokine storm' [38]. The increase in circulating CD4 T cells secreting higher levels of IFN- $\gamma$ in response to MTB antigens was also demonstrated by enzyme-linked immunospot assays or whole-blood IFN- $\gamma$ release assays [44,47]. The Elliot et al. [47] study similarly demonstrated increased levels of $\mathrm{T}$ cells reactive to purified protein derivative (PPD) and higher levels of IFN- $\gamma$ at IRIS event in a prospective cohort [47].

Restoration of TST reactivity (PPD reaction) in IRIS clearly pointed to an augmented Th1 response by multifunctional CD4 effector memory cells and an amplification of a particular subset of KIR-V $\delta 2+$ TCR $\gamma \delta$ T cells in IRIS patients, with a rejunuvated activity of APCs (CD11c+CD123-myeloid DC) compared with non-IRIS counterparts [42]. Bourgarit et al. [38] described it as a 'cytokine storm' unleashed by activated $\mathrm{T}$ cells during TB-IRIS. Reversal of defects in circulating DC of both myeloid and plasmacytoid origin also occurred after HAART and ATT. The early response to TB is predominated by neutrophils and TCR $\gamma \delta$ T cells that produce IFN- $\gamma$ by MHC-I ligand receptors of the NK family. TCR $\gamma \delta$ cells with KIR+ and NKR lectins like CD94/NKG2 were lower in IRIS patients throughout compared with non-IRIS patients who were specific for TB antigens [42].

\section{Cytokines}

During inflammation, these soluble mediators diffuse into the blood stream by spillover from the site of disease. Elevated cytokine production is directly related to the extent of initial lesion and severity of IRIS [56]. Early data from two studies have suggested that during IRIS, there is a shift of balance in favor of Th1 response, with the release of cytokines like IL-2, IL-12 and IFN- $\gamma$, IL- 6 and TNF- $\alpha$ over Th2 type [38,57]. Increased production of IL-18 by activated macrophages and higher levels of CXCL10 recruiting activated $\mathrm{T}$ effector cells together with a deficiency of 
CCL2 promoted IRIS occurrence [58]. In the prospective study by Bourgarit et al. [38], it was found that irrespective of differences in baseline levels of CD4, viral load, time of ART or IFN- $\gamma$ specific to PPD antigen prior to ART, there was a steep rise in the number of PPD-specific $\mathrm{T}$ cells producing IFN- $\gamma$ (identified by enzyme linked immunospot) during IRIS event, with a shift toward Th1 response (with higher levels of IL-2, IL-12, IP-10 and MIG), without a corresponding increase in Th2 response. This was seen in both stimulated and unstimulated cells. $\mathrm{T}$ cell activation marker expression (HLA-DR) was also pronounced in the IRIS group. Peaking of IFN- $\gamma$ production from PPD-specific $\mathrm{T}$ cells was preserved as well as sustained prospectively. In addition, the study showed that the response was not observed with ESAT-6 antigen. They concluded that an overwhelming Th1 response over Th2 was responsible for TB granuloma formation and enlargement of foci even in non-HIV individuals on ATT. This observation justified the use of anti-inflammatory therapy in controlling IRIS $[38,42]$.

The study by Tadokera et al. [56], on a cohort of confirmed HIV coinfected TB patients (ART naïve at baseline), showed higher IL-2, IL-15 and TNF- $\alpha$ transcriptome among IRIS cases with IL-5 RNA being more in non-IRIS cases than in unstimulated assays. When stimulated by $\mathrm{H} 37 \mathrm{Rv}$ strain of Mtb, there was an excessive secretion of RNA coding for IL-1b, IL-2, IL-5, IL-6, IL-10, IL-13, IL-15, IL-17A, IFN- $\gamma$, GM-CSF and TNF with IL-6, IL-13, IL-17A and IFN- $\gamma$, which remained significant after Bonferroni correction for multiple comparisons. The tissue culture supernatant nearly mirrored the same picture. Serum levels of IL-6, IL-8, IL-10, IL-12p40, IFN- $\gamma$ and TNF- $\alpha$ were higher in IRIS group compared with controls, with TGF- $\beta$ showing the opposite trend. TNF- $\alpha$ and IL- 6 levels reversed with steroid therapy, but not IFN- $\gamma$. Peculiarly, the Th2 cytokines IL-5 and IL-13 were also increased. A similar mechanism of 'cytokine release syndrome' also operated in ARDS, graft-versus-host reaction and many systemic inflammatory response syndromes including sepsis. This study extended its observations, to confirm the reversal of the increased levels of cytokines after steroid administration, which coincided with amelioration of IRIS symptoms [56]. IL-17, an important proinflammatory marker, was markedly increased at IRIS event, mediated by a distinct longer living central memory cell subset of the Th17 system. IL-17 contributes to cold abscess formation by augmenting neutrophil chemotaxis. Circulating levels of IL-7 were found in all lymphopenic conditions, and it usually normalizes with CD4 cell restitution. IRIS patients had a higher level of IL-7 that persisted even after normalization of CD4 by HAART compared with nonIRIS counterparts. Tadokera et al. [56] demonstrated in vitro the consistent and markedly increased levels of TNF- $\alpha$, IFN- $\gamma$ and IL- 6 in TB-IRIS patients. On the contrary, Zaidi et al. [10] found no difference in Th1 or Th2 cytokine levels prior to ART initiation among IRIS and non-IRIS groups. IL-6 has been implicated as an additional factor in atherogenesis and hyperlipidemia in HIV [59]. Worsley et al. [60] showed an increase in IL-10, in addition to IL-6 and IFN- $\gamma$, among patients presenting with TB adenitis (as an IRIS manifestation). They concluded that the increase in IL-10 could be a compensatory response. Pulmonary TBIRIS patients had an increase in IL- 5 and TNF- $\alpha$ compared with controls, even though the increase in IFN- $\gamma$ alone assumed significance.

Role of tissue remodeling enzymes in IRIS

Activated matrix metalloproteinases (MMPs) are involved in tissue remodeling, granuloma formation, repair as well as modulation of immune responses. Excessive MMP production has been reported in malignancy, sarcoidosis, interstitial lung disease and pulmonary $\mathrm{TB}$ especially in cavity formation [61]. MMP gene expression and protein secretion in patients who developed paradoxical TB-IRIS and similar non-IRIS control patients were recently analyzed by Tadokera et al. [62]. The study found that the transcripts coding for MMP-3, MMP-7 and MMP-10 were increased in in vitro assays in IRIS patients compared with non-IRIS individuals. The corresponding levels of the proteins were higher in the supernatants of patients who responded to steroid therapy. MMP-7 was more consistently elevated compared with other MMPs at multiple time points and levels decreased after steroid administration [62]. The association of TB-IRIS with a distinct pattern of MMP gene, protein activation and modulation has been suggested by Tadokera et al. as a novel therapeutic target for IRIS management [62].

\section{Unmasking TB \& paradoxical TB-IRIS: do they represent different entities?}

Even though similarities exist between the two, important differences do exist at the microlevel. The cause of paradoxical IRIS is due to embedded or residual antigens that are usually nonviable, whereas in the unmasking type, it is due to viable, live pathogens capable of causing the disease if not treated [1,9,63]. Data from the Haddow et al. [64] study showed that the median time to onset of paradoxical TB IRIS was 15 days (7-21) compared with 21 days (7-52) in the unmasking variety. Paradoxical IRIS can occur despite lymphopenia, solely driven by changes in activated status of $\mathrm{T}$ cells, with rapid decline in viral load being the most significant risk factor. Low CD4 and high viral load predicted paradoxical IRIS but not unmasking IRIS. Presence of lymphadenopathy, low hemoglobin and elevated CRP levels proved to be risk factors for occurrence of unmasking IRIS. The study by Haddow et al. [64] evaluated circulating biomarkers, but not peripheral blood mononuclear cells, using appropriate controls for both types of TB-IRIS. This study showed lower levels of monocyte chemoattractant protein-1 or CCL2, IL-10 and higher levels of CRP/IL-10 ratio in paradoxical IRIS compared with controls. TNF- $\alpha$, CRP and CRP/IL-10 ratio were significantly higher in the unmasking variety at IRIS. Oliver et al. [58] performed 
a longitudinal analysis estimating the cytokines CCL2, CXCL10 (IFN- $\gamma$-inducible protein 10) and IL-18, produced by the innate system, which played a crucial role in mounting an immune response against MTB. At IRIS event, levels of IL-18 in the unmasking type and IFN- $\gamma$ and CXCL10 in the paradoxical TB-IRIS were higher. Correspondingly, CCL2 levels were lower in both the IRIS groups compared with respective controls. No such difference was observed with CXCL9 or CXCL8. IL-18, like IL-12, induces IFN- $\gamma$ production, which results in macrophage activation, leading to sudden upsurge of Th-1 responses. Ianello et al. hypothesized that a deficiency of the IL-18 antagonist namely IL-18-binding protein allowed unabated action of IL-18, which resulted in IRIS [65]. The levels of IL-18 were increased to a greater extent in unmasking IRIS than paradoxical IRIS, due to a pronounced increase in IFN- $\gamma$ response to region of difference-1 (RD-1) antigens derived from live bacilli/viable antigens. CXCL10, which regulated trafficking of effector cells, both NK and T cells, to sites of treated infection, was increased in the paradoxical type compared with the unmasking variety. CCL2, critical for monocyte infiltration of affected sites like pleura, being lower prior to ART initiation could result in stagnation of antigens. This led to defective antigenic clearance, thereby predisposing to TBIRIS after ART [58]. Patients who presented with unmasking TB-IRIS had a more pronounced IFN- $\boldsymbol{\gamma}$ release response to RD-1 antigens (ESAT-6, TB7.7 and CFP-10) as well as to PPD than those without unmasking IRIS, after correction for pre-ART IFN- $\gamma$ levels, in the study by Elliot et al. [47]. The above facts support the hypothesis that viable antigens are involved in unmasking IRIS as RD-1 antigens can be released from living bacilli only [47].

\section{Predicting TB-IRIS}

The morbidity and clinical dilemma imposed by IRIS occurrence demands studying this phenomenon in greater detail in order to predict IRIS, thereby enabling prompt intervention (Figure 2). Mortality has been as high as $13 \%$ [18]. Needless to say, in order for a risk factor to function as a reliable predictor, it needs to be differentially expressed before ART or soon after ART, but importantly before occurrence of IRIS. Preliminary knowledge derived from retrospective cohorts and longitudinal studies found advanced stage of the HIV disease, coupled with presence of antigen dead or alive, to be the most potent clinical predictor for IRIS. Accordingly, low CD4 count [13,30,64], low BMI [24], low hemoglobin [30], high viral load [64], disseminated TB [4,5], extra pulmonary focus $[7,16,30]$ and negative TST [30] pre-ART, proved to be useful and simple predictors at baseline, with the only modifiable risk factor being ATTART interval. Shorter ATT-ART interval coupled with shorter duration of TB treatment at ART initiation is a credible predictor, which provides a conducive environment for IRIS occurrence [4,5,21]. The Michailides et al. study, however, did not show the above trend [66]. This makes thorough screening and adequate treatment of OI before initiation of HAART mandatory in IRIS prevention [29]. Breton et al. [16] found baseline $\mathrm{CD} 4 \%$ and increased ratio of $\mathrm{CD} 4 / \mathrm{CD} 8$ to be independent predictors of TB-IRIS. Absolute count of naive CD8+ cells was higher in TB-IRIS patients prior to ART who remained significant till 39 weeks post-ART [44]. Urinary lipoarbinomannan is not only useful in diagnosis of disseminated TB, but also in predicting IRIS [67]. Use of boosted protease inhibitors [15] was a predictor in one study, probably due to potent reduction in viral load. However, IRIS is not related to any specific ART regimen.

Probing into cellular and molecular levels for IRIS prediction, the study by Pean et al. [54] showed higher levels of NK cell degranulation (CD107a+), with increased expression of CD69 before ART initiation, to be a predictor of IRIS. Mahnke et al. [45] study on IRIS (of all OIs) showed increased frequencies of PD-1 cells of CD4+ and CD8+ origin before ART among IRIS cases. Antonelli et al. [43] found a higher frequency of exhausted Tregs (CD25+Foxp3 $\left.+\mathrm{CD} 127^{\mathrm{lo}}+\mathrm{PD}-1+\mathrm{Ki} 67+\right)$ and $\mathrm{CD} 4+$ and $\mathrm{CD} 8+\mathrm{T}$ cells that coexpressed CTLA4, LAG3 and ICOS before ART initiation, along with higher levels of IL-17. All the other cytokines and coreceptors showed no difference to be selected as a predictor. The study by Bourgarit et al. [42] found tuberculinspecific polyfunctional effector $\mathrm{T}$ cells and an amplification of KIR-V- $\delta 2+T C R \gamma \delta$ cells T cells, which could be a potential predictor of TB-IRIS. Oliver et al. [58] found lower CCL2 and higher levels of IL-18 and CXCL10, which could successfully predict IRIS and the prediction capacity increased on combining them with IFN- $\gamma$ responses to PPD and RD-1 antigen.

Pre-ART IFN- $\gamma$ responses to PPD and RD-1 were higher among IRIS patients in the receiver operator curves modeled with pre-ART levels of CCL2, CXCL10 and IL-18. Elliot $e t$ al. found significantly higher IFN- $\gamma$ levels in response to PPD and RD-1 antigens (after correction for pre-ART CD4 counts) in the TB-IRIS group enabling prediction, concordant with the TST response. The usefulness of IFN- $\gamma$ release assays in predicting IRIS, especially unmasking IRIS, was made possible by the longitudinal design of the study, computing levels at multiple time points. Furthermore, they were able to stratify the risk of IRIS based on the levels of IFN- $\gamma$ production paralleling the skin response by TST. The Haddow et al.'s [64] study selected patients who had an uneventful immune recovery (without TB or IRIS) as controls instead of healthy individuals because the latter had a totally different $\mathrm{T}$ cell homeostasis. They found no good predictor in the paradoxical IRIS group, but CRP and IFN- $\gamma$ levels were higher in the unmasking variety, which could serve as possible predictors. When the combined observations in the two IRIS groups were compared with the non-IRIS group (after adjustment for CD4, high viral load, presence of TB), lower IL-2 concentrations and higher CRP at baseline became significant predictors of IRIS. Since the increase of IFN- $\gamma$ and CRP were fourfold rise in unmasking IRIS, which closely paralleled the level of cytokines in an undiagnosed or subclinical TB patient, differentiation of undiagnosed or subclinical 


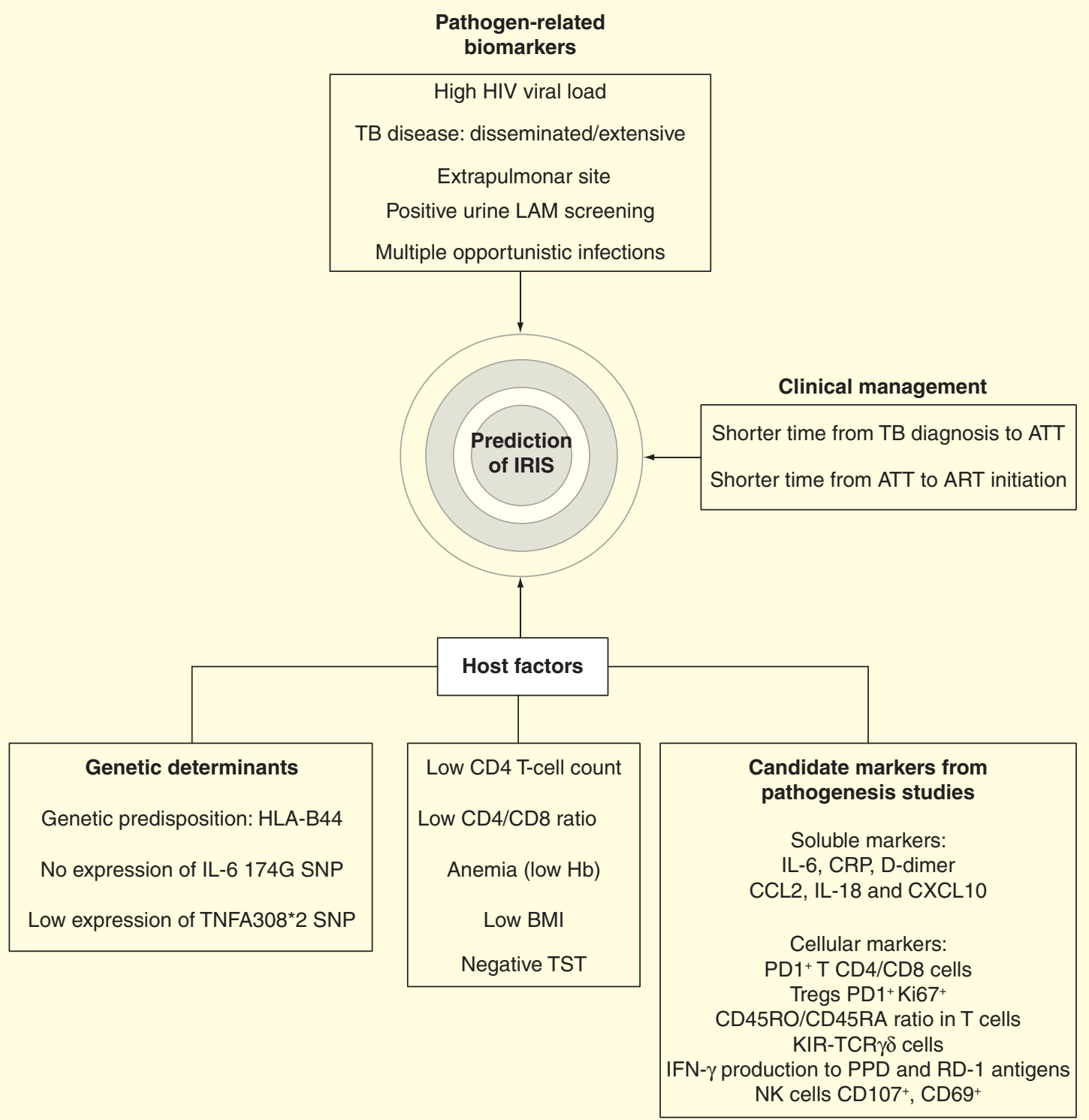

Figure 2. Risk factors that reliably predict immune reconstitution inflammatory syndrome.

ART: Antiretroviral therapy; ATT: Anti-TB therapy; Hb: Hemaglobin; HLA: Human leukocyte antigen; IRIS: Immune reconstitution inflammatory syndrome; LAM: Lipoarabinomannan; SNP: Single nucleotide polymorphism; TB: Tuberculosis; TST: Tuberculin skin test.

infection from unmasking IRIS still remains a herculean task [29,64].

Our prospective TB-IRIS study done on a pure cohort of HIV-positive ART-naive patients with culture-positive rifampicin-sensitive pulmonary TB found the sensitivity for IRIS prediction to be $80 \%$ for IL- 6 and $76 \%$ for CRP, which increased to $92 \%$ when both factors were combined. All other cytokines were similar at baseline between the IRIS cases and others [4]. Porter et al. [68] found D-Dimer, CRP and IL-6 to be predictors of not only IRIS (including TB-IRIS) but also mortality and disease progression. IRIS-B occurred more often in patients carrying homozygous genes at the TNF- $\alpha{ }^{*} 308$ loci and who did not carry IL-6-174*C. HLA-B44 has been found to predispose to IRIS, but considerable ethnic and regional variations exist in HLA evaluation [31].

\section{Current remedies for IRIS}

Meticulous screening of OIs pre-ART and optimizing timing of ART remains the best options for avoiding IRIS [5,29]. Antiinflammatory agents form the backbone of IRIS management, ranging from nonsteroidal anti-inflammatory drugs like ibuprofen to parenteral steroids and thalidomide in refractory cases [69,70]. Steroids are nonspecific inhibitors of inflammatory response, quite effective in IRIS [71]. In our cohort, steroids were required in $85 \%$ of the cases [4]. Meintjes et al. demonstrated the reduction in severity of IRIS after steroid administration that correlated with the decrease in levels of cytokines 
like IL-6, CRP, IL-10, IL-12, IP-10 and TNF- $\alpha$ [71]. The fact that corticosteroids ameliorate IRIS, partly by decreasing the concentration of proinflammtory cytokines, could pave the way for use of targeted anti-inflammatory therapy like tocilizumab, an IL-6 blocker [72].

The increase in eicosanoids and leucotrienes during IRIS could be blocked by antagonists like montelukast, but use in IRIS is supported only by anecdotal reports [73]. Statins are known to reduce inflammatory cell recruitment partly by reducing CXCL10 and modulation of IL-6 pathways [74]. CXCR3 antagonists, the main receptor for CXCL10, are also being explored for IRIS prevention [75]. Effector $\mathrm{T}$ cells also expressing CCR5 and CCR5 chemokine inhibitors like Maraviroc have been found to reduce inflammation possibly by retarding migration of these cells to the site of inflammation and peripheral tissues. There was a reduction in D-dimer levels as well as CRP in the Maraviroc arm compared with efavirenz arm in the study by Funderburg et al [76]. However, the CADIRIS trial, comparing Maraviroc versus placebo in a multiarm randomized controlled clinical trial done in South Africa, failed to prove the beneficial effect of Maraviroc in preventing IRIS $(22.8 \%$ in Maraviroc vs $23.6 \%$ in placebo arm, $\mathrm{p}=0.66 \mathrm{~ns})$ [77]. Certain novel mechanisms for blocking IRIS appear promising (theoretical at present) but require further scientific exploration. Anti-CD28 therapy (TGN1412) could be an alternative, as in HINI treatment of ARDS, but it causes an acute illness [78]. TNF- $\alpha$ is an important mediator of inflammation including fever. Blockade could reverse the symptoms but can also promote relapse by interfering with MTB-specific immune responses. MMP-7 activity and other MMPs may also represent therapeutic targets for reducing severity of paradoxical TB-IRIS [62]. The use of these immunomodulators, even if found to be effective, should be restricted to severe forms like neurological and abdominal IRIS [2,79].

\section{Expert commentary}

Our review summarizes the recent developments and current scientific concepts regarding TB-IRIS and the role played by the immunological cells and soluble markers, both individually and in combination. Even though IRIS is associated with a mortality of only $3 \%$, its morbidity and potential clinical complications mandate utilization of tertiary care to a great extent. A collaborative medical effort to manage IRIS involving experts from various medical fields, incur a huge cost. This poses a critical challenge in the path of decentralization of the ART program, especially for HIV-TB coinfected patients with advanced disease, which formed the most vulnerable population to IRIS. The possibility of finding reliable biomarkers for prediction or diagnosis is not far away especially in the light of recent advances in immunological studies exploring many new pathways and immune mediators responsible for IRIS occurrence. Once the knowledge about the role of these mediators is well established, then drugs that selectively inhibit these inflammatory pathways would follow suit. These drugs, if found to be effective in inhibiting/blocking specific inflammatory targets, will help in efficiently preventing IRIS without the need for use of broad-spectrum anti-inflammatory agents like steroids, paving the way for an uneventful recovery of the immune system. The prevention and successful management of IRIS assume immense importance in the wake of preservation of adherence to the lifelong therapy of ART.

\section{Five-year view}

\section{Frontiers to be explored \& conquered}

IRIS is still a mystery with a partially unraveled mechanism of occurrence. Foretelling the occurrence of IRIS is still far from reality, due to pleiotropism of cytokines and effector cells, which can be polyfunctional. Since cytokines are produced by multiple cells, determining the cytokines that have lesser pleiotropism and redundancy could prove to be better targets for therapeutic exploration. They could function as valid predictors as well. Oxidative stress at the cellular level needs to be investigated further. The role of Treg should be focused in depth to support natural mechanisms against IRIS. Blockade of IFN- $\gamma$ production by antagonists including IL-18-binding protein could be a novel idea [65]. IL-1RA and IL-6 antagonists are promising candidates in IRIS management in the near future. IL-10, a Th-2 cytokine, could be induced to counteract Th1 responses due to its anti-inflammatory effect. Due to the complexity of disease manifestation and pathogenic mechanisms involved in IRIS, several challenges need to be addressed in order to achieve a scenario in which IRIS can be predicted with greater confidence and clinical management optimized.

\section{Acknowledgements}

The authors wish to thank I Sereti, L Antonelli, A Sher, BO Porter, T Nutman from the National Institute of Health, USA, S Babu ICER facility NIRT, C Chandrasekhar, Superintendant, OR Krishnarajasekar, Deputy Superintendant, S Kumar, K Raja, N Ravichandran, Government Hospital of Thoracic Medicine, Tambaram Sanatorium. R Sridhar, Prof. Chest medicine, Stanley Medical College, Chennai. K Bhanu, Professor of neurology, S Sekhar, ART Medical Officer, Government Rajiv Gandhi Hospital, Chennai, C Padmapriyadarsini, PA Menon, PK Bhavani, K Vijay, S Devarajulu Reddy, from the National Institute for Research in Tuberculosis Chennai for their valuable inputs, the staff of IRIS study team S Subramanyam, LE Hanna, S Anbalagan, S Rajasekaran, $N$ Logeswaran, A Stella Mary, $N$ Hariharan for their technical help and $N$ Santhanakumar for his excellent secretarial assistance.

\section{Financial \& competing interests disclosure}

The authors have no relevant affiliations or financial involvement with any organization or entity with a financial interest in or financial conflict with the subject matter or materials discussed in the manuscript. This includes employment, consultancies, honoraria, stock ownership or options, expert testimony, grants or patents received or pending, or royalties.

No writing assistance was utilized in the production of this manuscript. 


\section{Key issues}

- Early initiation of antiretroviral therapy (ART) with active Mycobacterium tuberculosis, a chronic infection with persisting antigens, sets an ideal stage for immune reconstitution inflammatory syndrome (IRIS).

- Dysregulated augmentation of immune responses after initiation of highly active ART (HAART) despite effective suppression of plasma viremia could lead to clinical deterioration.

- Restoration of pathogen-specific immune responses to either viable, active pathogens or residual antigens causes unmasking or paradoxical IRIS that usually occurs within 3 months after HAART initiation.

- Abrupt rise in CD4 T-cell count or rapid decline in viral load contributes to IRIS, but considerable variation exists in the reconstitution of CD4 lymphocytes upon HAART initiation questioning their role as reliable predictors.

- The abrupt rise in nonspecific mediators like IFN- $\gamma$ levels in response to purified protein derivative and region of difference- 1 is much more striking in unmasking than paradoxical IRIS.

- Increase in activated CD4 T cells induces an explosive exaggerated Th1 type of cytokine response overriding Th2 response, culminating in IRIS, justifying use of anti-inflammatory drugs.

- Matrix metalloproteinases have been recently implicated in tuberculosis (TB)-IRIS.

- Low CD4, low BMI, low hemoglobin, high viral load, disseminated TB, extra pulmonary foci and negative tuberculin skin testing pre-ART are useful clinical predictors, with the only modifiable risk factor being time to ART initiation.

- Our prospective TB-IRIS study in India, on a cohort of HIV-positive patients with culture-positive rifampicin-sensitive pulmonary TB, found the combined predictive value of IL-6 and CRP to be $92 \%$ in paradoxical IRIS.

- Specific molecular targets would be useful to precisely control the phenomenon without disturbing all the constituents of restored immunity.

\section{References}

Papers of special note have been highlighted as:

- of interest

•• of considerable interest

1. Lawn SD, Lipman MC, Easterbrook PJ. Immune reconstitution disease associated with Mycobacterial infections. Curr Opin HIV AIDS 2008;3:425-31

2. Muller M, Simon W, Colebunders R, et al. Immune reconstitution inflammatory syndrome in patients starting antiretroviral therapy for HIV infection: a systemic review and meta analysis. Lancet Infect Dis 2010;10:251-61

3. Shelburne SA, Hamill RJ, Rodriguez-Barradas MC, et al. Immune reconstitution inflammatory syndrome: emergence of a unique syndrome during highly active antiretroviral therapy. Medicine (Baltimore) 2002;81(3): 213-27

4. Narendran G, Andrade BB, Porter BO, et al. Paradoxical tuberculosis immune reconstitution inflammatory syndrome (TB-IRIS) in HIV patients with culture confirmed pulmonary tuberculosis in India and the potential role of IL- 6 in prediction. PLoS One 2013;8(5):e63541

-• Found that IL-6, even though a nonspecific inflammatory cytokine, still has a key role to play in prediction especially when combined with CRP.
5. Meintjes G, Lawn SD, Scano F, et al. Tuberculosis-associated immune reconstitution inflammatory syndrome: case definitions for use in resource-limited settings. Lancet Infect Dis 2008;8(8):516-23

- A detailed and succinct description of classification useful in diagnosing immune reconstitution inflammatory syndrome (IRIS) even in resource-limited settings.

6. Murdoch DM, Venter WD, Feldman C, et al. Incidence and risk factors for the immune reconstitution inflammatory syndrome in HIV patients in South Africa: a prospective study. AIDS 2008;22(5): 601-10

7. Breen RA, Smith CJ, Bettinson H, et al. Paradoxical reactions during tuberculosis treatment in patients with and without HIV co-infection. Thorax 2004;59:704-7

8. Narita M, Ashkin D, Hollender ES, et al. Paradoxical worsening of tuberculosis following antiretroviral therapy in patients with AIDS. Am J Respir Crit Care Med 1998;158:157-61

9. Sereti I, Rodger AJ, French MA, et al. Biomarkers in immune reconstitution inflammatory syndrome: signals from pathogenesis. Curr Opin HIV AIDS 2010; 5(6):504-10

- Found that both innate and adaptive immunity have their respective roles to play in IRIS, which varies with the underlying pathogen.

10. Zaidi I, Peterson K, Jeffries D, et al. Immune reconstitution inflammatory syndrome and the influence of $\mathrm{T}$ regulatory cells: a cohort study in The Gambia. PLoS One 2012;7(6):e39213

11. Barber DL, Bruno AB, Sereti I, et al. Immune reconstitution inflammatory syndrome: the trouble with immunity when you had none. Nature 2012;10:150-6

- Suggested a common pathway that follows immunosuppression followed by immune recovery that could explain IRIS using the mouse model.

12. Pepper DJ, Marais S, Maartens G, et al. Neurologic manifestations of paradoxical tuberculosis-associated immune reconstitution inflammatory syndrome: a case series. Clin Infect Dis 2009;48:96-107

13. French MA, Price P, Stone SF, et al. Immune restoration disease after antiretroviral therapy. AIDS 2004;18: 1615-27

- The wide spectrum of clinical manifestations of IRIS explained in detail.

14. Colebunders R, John L, Huyst V, et al. Tuberculosis immune reconstitution inflammatory syndrome in countries with limited resources. Int J Tuberc Lung Dis 2006;10(9):946-53 
15. Manabe YC, Campbell JD, Sydnor E, et al. Immune reconstitution inflammatory syndrome: risk factors and treatment implications. J Acquir Immune Defic Syndr 2007;46(4):456-62

16. Breton G, Duval X, Estellat C, et al. Determinants of immune reconstitution inflammatory syndrome in HIV type 1-infected patients with tuberculosis after initiation of antiretroviral therapy. Clin Infect Dis 2004;39:1709-12

17. Narendran G, Swaminathan S. Tuberculosis immune reconstitution inflammatory syndrome: profile of an enigmatic condition. Curr Sci 2013;105(5):657-65

18. Marais S, Meintjes G, Pepper DJ, et al. Frequency, severity, and prediction of tuberculous meningitis immune reconstitution inflammatory syndrome. Clin Infect Dis 2013;56(3):450-60

19. Buckingham SJ, Haddow LJ, Shaw PJ, et al. Immune reconstitution inflammatory syndrome in HIV-infected patients with mycobacterial infections starting highly active anti-retroviral therapy. Clin Radiol 2004;59(6):505-13

20. Swaminathan S, Padmapriyadarsini C, Venkatesan P, et al. Efficacy and safety of once-daily nevirapine-or efavirenz-based antiretroviral therapy in hiv associated tuberculosis: a randomized clinical trial. Clin Infect Dis 2011;53(7):716-24

21. Lawn SD, Myer L, Bekker LG, et al. Tuberculosis-associated immune reconstitution disease: incidence, risk factors and impact in an antiretroviral treatment service in South Africa. AIDS 2007;21: 335-41

22. Lawn SD, Macallan DC. Hypercalcemia: a manifestation of immune reconstitution complicating tuberculosis in an HIVinfected person. Clin Infect Dis 2004;38(1): 154-5

23. Price P, Murdoch DM, Agarwal U, et al. Immune restoration diseases reflect diverse immunopathological mechanisms. Clin Microbiol Rev 2009;22(4):651-63

24. Letang E, Miro JM, Nhampossa T, et al. Incidence and predictors of immune reconstitution inflammatory syndrome in a rural area of Mozambique. PLoS One 2011; 6(2):e16946

25. Abdool Karim SS, Naidoo K, Grobler A, et al. Integration of antiretroviral therapy with tuberculosis treatment. N Engl J Med 2011;365(16):1492-501

26. Blanc FX, Sok T, Laureillard D, et al. Earlier versus later start of antiretroviral therapy in HIV-infected adults with tuberculosis. N Engl J Med 2011;365(16): 1471-81

27. Haddow LJ, Moosa MY, Mosam A, et al. Incidence, clinical spectrum, risk factors and impact of HIV-associated immune reconstitution inflammatory syndrome in South Africa. PLoS One 2012;7(11):e40623

28. Ratnam I, Chiu C, Kandala NB, et al. Incidence and risk factors for immune reconstitution inflammatory syndrome in an ethnically diverse HIV type-1 infected cohort. Clin Infect Dis 2006;42:418-27

29. Lawn SD, Meintjes G. Pathogenesis and prevention of immune reconstitution disease during antiretroviral therapy. Expert Rev Anti Infect Ther 2011;9(4):415-30

-• Preventive aspects of IRIS are dealt with.

30. Worodria W, Menten J, Massinga-Loembe $\mathrm{M}$, et al. Clinical spectrum, risk factors and outcome of immune reconstitution inflammatory syndrome in patients with tuberculosis-HIV co-infection. Antivir Ther 2012;17(5):841-8

31. Price P, Morahan G, Huang D, et al. Polymorphisms in cytokine genes define subpopulations of HIV-1 patients who experienced immune restoration diseases. AIDS 2002;16:2043-7

32. Abdool Karim SS, Naidoo K, Grobler A, et al. Timing of initiation of antiretroviral drugs during tuberculosis therapy. N Engl J Med 2010;362(8):697-706

33. Havlir DV, Kendall MA, Ive P, et al. Timing of antiretroviral therapy for HIV-1 infection and tuberculosis. N Engl J Med 2011;365(16):1482-91

- A clear description of benefits of antiretroviral therapy using composite response stratified based on CD4.

34. Velasco M, Castilla V, Sanz J, et al. Effect of simultaneous use of highly active antiretroviral therapy on survival of HIV patients with tuberculosis. J Acquir Immune Defic Syndr 2009;50(2):148-52

35. Narendran G, Swaminathan S. Comparing daily vs intermittent regimen of ATT in HIV with pulmonary tuberculosis. 2009. Available from: http://clinicaltrials.gov/ct2/ show/NCT00933790?term=Daily+Vs +intermittent+ATT+in+HIV\&rank=1 [Last accessed 25 January 2014]

36. Revised NACO guidelines for ART initiation in adults. Office memorandum T-11020/36/2005 [Last accessed 4 November 2011]

37. WHO. Rapid advice: antiretroviral therapy for HIV infection in adults and adolescents. November 2009. Available from: www.who. int/hiv/pub/arv/rapid_advice_art.pdf?ua= 1 [Last accessed 25 November 2013]

38. Bourgarit A, Carcelain G, Martinez V, et al. Explosion of tuberculin-specific Th1responses induces immune restoration syndrome in tuberculosis and HIV co-infected patients. AIDS 2006;20(2):F1-7

-• Overriding Th1 response as a cause of IRIS after adjustment of CD4 and viral load paved way for treatment strategies in future.

39. Battegay M, Nuesch R, Hirschel B, et al. Kaufmann GR. Immunological recovery and antiretroviral therapy in HIV-1 infection. Lancet Infect Dis 2006;6(5):280-7

40. Renaud M, et al. Determinants of paradoxical CD4 cell reconstitution after protease inhibitor-containing antiretroviral regimen. AIDS 1999;13:669-76

41. Lange CG, Lederman MM. Immune reconstitution with antiretroviral therapies in chronic HIV-1 infection. J Antimicrob Chemother 2003;51:1-4

42. Bourgarit A, Carcelain G, Samri A, et al. Tuberculosis-associated immune restoration syndrome in HIV-1-infected patients involves tuberculin-specific CD4 Th1 cells and KIR-negative gamma delta $\mathrm{T}$ cells. J Immunol 2009;183(6):3915-23

-• Higher proportion of patients experiencing IRIS exhibits gamma delta $T$ cells lacking KIR expression compared with non-IRIS counterparts.

43. Antonelli LR, Mahnke Y, Hodge JN, et al. Elevated frequencies of highly activated CD4+ T cells in HIV+ patients developing immune reconstitution inflammatory syndrome. Blood 2010;116(19): 3818-27

- Activated replicative effector memory cells play a central role in IRIS causation with higher secretion of IFN- $\gamma$ and IL-7.

44. Espinosa E, Romero-Rodriguez DP Cantoral-Diaz MT, et al. Transient expansion of activated CD8+ T cells characterizes tuberculosis-associated immune reconstitution inflammatory syndrome in patients with HIV: a case control study. J Inflamm (Lond) 2013;10(1):21

45. Mahnke YD, Greenwald JH, DerSimonian R, et al. Selective expansion of poly functional pathogen-specific $\mathrm{CD} 4(+)$ $T$ cells in HIV-1-infected patients with immune reconstitution inflammatory syndrome. Blood 2012;119(13):3105-12

-• Robust increase in pre-existing polyfunctional effector $T$ cells that 
specifically target the underlying coinfection representing a dysregulated immune response occurs in IRIS.

46. Eggena MP, Barughare B, Jones N, et al. depletion of regulatory $\mathrm{T}$ cells in HIV infection. J Immunol 2005;174:4407-14

47. Elliott JH, Vohith K, Saramony S, et al. Immunopathogenesis and diagnosis of tuberculosis and tuberculosis-associated immune reconstitution inflammatory syndrome during early antiretroviral therapy. J Infect Dis 2009;200(11):1736-45

48. Montes M, Cesar S, Dororthy EL. Normalization of Fox P3+regulatory cells in response to effective antiretro viral therapy. J Infect Dis 2011;203:496-9

49. Meintjes G, Wilkinson KA, Rangaka MX, et al. Type 1 helper $\mathrm{T}$ cells and FoxP3positive $\mathrm{T}$ cells in HIV-tuberculosisassociated immune reconstitution inflammatory syndrome. Am J Respir Crit Care Med 2008;178(10):1083-9

- Studied the proportion of T helper and Treg longitudinally and found them to be equivalent with the non-IRIS group.

50. Seddiki N, Sasson SC, Santner-Nanan B, et al. Proliferation of weakly suppressive regulatory $\mathrm{CD} 4+\mathrm{T}$ cells is associated with over-active CD4+ T-cell responses in HIV-positive patients with mycobacterial immune restoration disease. Eur J Immunol 2009;39(2):391-403

- Clear explanation of the role of Treg and their defective function despite their increasing numbers.

51. Dsouza M, Fontenot AP, Mack DG, et al. Programmed death 1 expression on HIVspecific CD4 T cells is driven by viral replication and associated $\mathrm{T}$ cell dysfunction. J Immunol 2007;179(3):1979-87

52. Stewart CA, Vivier E, Colonna M. Strategies of natural killer cell recognition and signaling. Curr Top Microbiol Immunol 2006;298:1-21

53. Mavillo D, Benjamin J, Daucher, et al. Natural killer cells in HIV-infcetion; dichotomus effects of viraemia o ninhibitory and activating receptors and their function correlates. Proc Natl Acad Sci USA 2003; 100(25):15011-16

54. Pean P, Nerrienet E, Madec $\mathrm{Y}$, et al. Natural killer cell degranulation capacity predicts early onset of the immune reconstitution inflammatory syndrome (IRIS) in HIV-infected patients with tuberculosis. Blood 2012;119(14):3315-20

55. Clifford DB, De Luca A, Simpson DM, et al. Natalizumab-associated progressive multifocal leukoencephalopathy in patients with multiple sclerosis: lessons from 28 cases. Lancet Neurol 2010;9(4): 438-46

56. Tadokera R, Meintjes G, Skolimowska KH, et al. Hypercytokinaemia accompanies HIV-tuberculosis immune reconstitution inflammatory syndrome. Eur Respir J 2011; 37(5):1248-59

- Showed not only that Th-1 response was intense at IRIS caused by cytokine storm but also showed the reversal in levels of inflammatory cytokines with corresponding amelioration of symptoms with anti-inflammatory therapy.

57. Tan DB, Yong YK, Tan HY, et al. Immunological profiles of immune restoration disease presenting as mycobacterial lymphadenitis and cryptococcal meningitis. HIV Med 2008; 9(5):307-16

58. Oliver BG, Elliott JH, Price P, et al. Mediators of innate and adaptive immune responses differentially affect immune restoration disease associated with Mycobacterium tuberculosis in HIV patients beginning antiretroviral therapy. J Infect Dis 2010;202(11):1728-37

59. Gierens $H$, Nauck $M$, Roth $M$, et al. Interleukin-6 stimulated DL receptor gene expression via activation of sterol-responsive and Sp 1 binding elements. Arterioscler Thromb Vasc Biol 2000;20:1777-83

60. Worsley CM, Suchard MS, Stevens WS et al. Multi-analyte profiling of ten cytokines in South African HIV-infected patients with immune reconstitution inflammatory syndrome (IRIS). AIDS Res Ther 2010;7:36

61. Volkman HE, Pozos TC, Zheng J, et al. Tuberculous granuloma induction via interaction of a bacterial secreted protein with host epithelium. Science 2010;327: 466-9

62. Tadokera R, Meintjes G, Wilkinon KA, et al. Matrix metalloproteinases and tissue damage in HIV-tuberculosis immune reconstitution inflammatory syndrome. Eur J Immunol 2014;44(1):127-36

63. Manabe YC, Breen RAM, Perti T, et al. et al. Unmasking tuberculosis and tuberculosis immune reconstitution inflammatory disease: a disease spectrum after initiation of antiretroviral therapy. J Infect Dis 2009;199:437-44

64. Haddow LJ, Dibben O, Moosa MY, et al Circulating inflammatory biomarkers can predict and characterize tuberculosis-associated immune reconstitution inflammatory syndrome. AIDS 2011;25(9):1163-74

- Segregated analysis of predictors for both unmasking and paradoxical IRIS.

65. Ianello A, Boulassel MR, Samarani S, et al. HIV-1 causes imbalance in the production of interleukin-18 and its natural antagonist in HIV infected individuals; implications of enhanced viral replication. J Infect Dis 2010;201:608-17

66. Michailides C, Pozniak AL, Mandalia S, et al. Clinical characteristics of IRIS syndrome in patients with HIV and tuberculosis. Antivir Ther 2005;10:417-22

67. Lawn SD, Edwards DJ, Kranzer K, et al. Urine Lipoarabinamannan assay for tuberculosis screening before antiretroviral therapy diagnostic yield and association with immune reconstitution disease. AIDS 2009;23:1875-80

68. Porter BO, Ouedraogo GL, Hodge JN, et al. D-dimer and CRP levels are elevated prior to antiretroviral treatment in patients who develop IRIS. Clin Immunol 2010; 136(1):42-50

69. Meintjes G, Wilkinson RJ, Morroni C et al. Randomized placebo-controlled trial of prednisone for paradoxical tuberculosis-associated immune reconstitution inflammatory syndrome. AIDS 2010;24(15):2381-90

-• Provided concrete evidence for the use of steroids for IRIS that reduced duration of stay in hospital without compromising on survival.

70. Brunel AS, Reynes J, Tuaillon E, et al. Thalidomide for steroid-dependent immune reconstitution inflammatory syndromes during AIDS. AIDS 2012;26(16):2110-12

71. Meintjes G, Skolimowska KH,

Wilkinson KA, et al.

Corticosteroid-modulated immune activation in the tuberculosis immune reconstitution inflammatory syndrome. Am J Respir Crit Care Med 2012;186(4): 369-77

72. Hennigan S, Kavanaugh A. Interleukin- 6 inhibitors in the treatment of rheumatoid arthritis. Ther Clin Risk Manag 2008;4:767-75

73. Hardwick C, White D, Morris E, et al. Montelukast in the treatment of HIV associated immune reconstitution disease. Sex Transm Infect 2006;82:513-14

74. Sun HY, Singh N. Potential role of statins for the management of immune reconstitution syndrome. Med Hypotheses 2010;76(3):307-10 
75. Knight RL, Allen DR, Birch HL, et al. Development of CXCR3 antagonists. Part 4: discovery of 2-amino-(4-tropinyl) quinolines. Bioorg Med Chem Lett 2008;18:629-33

76. Funderburg N, Kalinowska M, Eason J, et al. Effects of Mraviroc and Efavirenz on markers of immune activation and inflammation and associations with CD4+ cells rise in HIV infected patients. PLoS One 2010;5(10):e13188

77. Sierra-Madero JG, et al. Maraviroc does not prevent IRIS in high risk subjects initiating
ART. (CADIRIS Trial) Abstract 182LB. 20th Conference on retrovirus and opportunistic infections. 2013.Available from: www.hivandhepatitis.com/hiv-aids/hivaids-topics/hiv-prevention/4050-croi-2013maraviroc-does-not-prevent-immunereconstitution-syndrome [Last accessed 30 January 2014]

78. Suntharalingam G, Perry MR, Ward S, et al. Cytokine storm in a phase 1 trial of the anti-CD28 monoclonal antibody TGN1412. N Engl J Med 2006;355(10): 1018-28
79. Marais S, Scholtz P, Pepper DJ, et al. Neuroradiological features of the tuberculosis-associated immune reconstitution inflammatory syndrome. Int J Tuberc Lung Dis 2010;14(2):188-96

80. Manosuthi W, Kiertiburanakul S, Phoorisri $\mathrm{T}$, et al. Immune reconstitution inflammatory syndrome of tuberculosis among HIV-infected patients receiving antituberculous and antiretroviral therapy. J Infect 2006;53(6):357-63 